\title{
Managing Uncertainty in Nursing - Newly Graduated Nurses' Experiences of Introduction to the Nursing Profession
}

\author{
Elina Ingvarsson, $B S c, R N^{1}$, Josefin Verho, $B S c, R N^{1}$ and Kristina Rosengren, $P h D, R N^{1,2^{*}}$ \\ ${ }^{1}$ Sahlgrenska Academy, Institute of Health and Care Sciences, University of Gothenburg, Sweden \\ ${ }^{2}$ Department of Internal Medicine, Sahlgrenska University Hospital, Mölndal, Sweden
}

*Corresponding author: Kristina Rosengren, PhD, RN, Associate Professor, Sahlgrenska Academy, Institute of Health and Care Sciences, University of Gothenburg; Department of Internal Medicine, Sahlgrenska University Hospital, Sweden, ORCID iD: 0000-0002-3221-2062

\begin{abstract}
Background: The introduction to nursing, which is the conversion of theoretical knowledge into practice skills in complex healthcare settings, is emphasized as difficult due to patient safety. Therefore, the aim of this study was to describe newly graduated nurses' experiences of introduction to nursing in a medical department (medical, emergency) at a university hospital in western Sweden.

Method: A manifest qualitative content analysis with an inductive approach was used based on five semistructured interviews with newly graduated nurses who work in a medical department at Sahlgrenska University Hospital in Gothenburg, Sweden.

Findings: One main category was found, managing uncertainty in the nursing profession, which includes three subcategories, namely, being humble as a newly graduated nurse, being adaptable as a newly graduated nurse and being a staff member instead of a nursing student. This category and these subcategories are described in the results.

Conclusion: The transition from education to registered nurse is a difficult step due to the anxiety that stems from making mistakes regarding limited experience related to the demands and requirements of the job. Therefore, standardized (routines, guidelines) and individualized (experiences) introductions facilitate patient safety. Moreover, person-centeredness ensures that every nurse is a capable person with resources to use in health care, i.e. a partnership within a healthy working environment that transforms novices to experts in nursing.
\end{abstract}

\section{Keywords}

Introduction, Mentorship, Newly graduated nurse, Supervision, Transition, Person-centeredness, Qualitative content analysis

\section{Introduction}

After a minimum of three years of university studies, newly graduated nurses are eager to start working. However, future nurses express anxiety about entering the nursing profession, which is why the introduction to the nursing profession is important to ensure patient safety $[1,2]$. The current study intends to broaden the knowledge regarding this introduction to improve newly graduated nurses' work environment and ensure a high quality of care.

\section{Background}

A nursing education develops knowledge regarding health and well-being and is an art that uses objective (quantitative) and subjective (qualitative) perspectives. Theoretical models prepare nursing students to understand context and behaviors. However, practical wisdom and tacit knowledge grows through experience, which is why it is difficult to learn in only a theoretical perspective $[3,4]$. Therefore, theoretical and practical knowledge are required to transform and translate nursing studies into clinical settings [5]. The opportunities for nursing students to participate in a peer mentoring relationship improve nursing, and mentorship helps students to fulfill future professional responsibilities by developing skills that improve communication and critical thinking [6]. Moreover, to facilitate future nurse's assignments in health care, educators in universities and clinical settings need innovative pathways that bring students and clinicians together as team

Citation: Ingvarsson E, Verho J, Rosengren K (2019) Managing Uncertainty in Nursing - Newly Graduated Nurses' Experiences of Introduction to the Nursing Profession. Int Arch Nurs Health Care 5:119. doi.org/10.23937/2469-5823/1510119

Accepted: March 23, 2019: Published: March 25, 2019

Copyright: (C) 2019 Ingvarsson E, et al. This is an open-access article distributed under the terms of the Creative Commons Attribution License, which permits unrestricted use, distribution, and reproduction in any medium, provided the original author and source are credited. 
members to overcome the gap between theory and practice [4]. Nurse's had several task, one is preventative care $[7,8]$; however, when more care is needed, nursing must be performed in collaboration according to the team's practical, empirical and personal knowledge $[4,9]$.

The complexity between theory and practice challenges newly graduated nurses. Entering the nursing profession is described as a reality shock, and theoretical education is difficult to address in a practical setting. Nurses acquire nursing knowledge and skills (knowing how) by learning the theory (knowing that) that is developed during nursing education and later in clinical practice according to a nurse's personality. This transition is described in the five stages of novice, advanced beginner, competent, proficient and expert [5]. Being transformative, integrative and representative, competences include support, confirmation, participation, and problem solving in the daily work as a nurse $[7,8]$. Moreover, a high quality of care focuses on patients' medical issues and their experiences, as well as resources regarding health and well-being [10]. Although nursing is unpredictable and demanding due to a variety of health problems and patients of all ages, teamwork is needed to provide a high quality of care [11]. According to the research [12], newly graduated nurses' experience emergency care as challenging, exciting and intimidating due to rapid changes with a high degree of problem solving. In addition, newly graduated nurses' experience anxiety and reduced self-confidence regarding their limited knowledge and skills; therefore, continuous supervisors and/or mentors are significant to the introduction to the nursing profession [13]. However, experienced nurses (supervisors) use different skills (tacit knowledge) in nursing, which can be confusing and can cause uncertainty among newly graduated nurses. Therefore, newly graduated nurses who work in a rapidly changing work environment need mentorship and long-term support that provide conditions to increase their self-confidence and independence $[6,14]$.

The expectations regarding newly graduated nurses' clinical skills and responsibility to handle patients contribute to occupational stress. Interprofessional teamwork is therefore emphasized to manage a high quality of care [15]. However, teamwork requires a healthy work environment [14], i.e., a supportive culture and opportunity for reflection at work $[13,16]$. Therefore, the transition from student to registered nurse is challenging as theoretical knowledge is translated into practical settings. For this reason, the current study intends to broaden the knowledge regarding the introduction to nursing.

\section{Aim}

The aim of this study was to describe newly graduated nurses' experience of introduction to a medical department (medical, emergency unit) at a university hospital in western Sweden.

\section{Method}

\section{Settings}

Sahlgrenska University Hospital [17] is one of the largest workplaces in the county council of Västra Götaland with approximately 16,000 employees who are divided into 120 departments and located at four different hospitals in the Gothenburg area, namely, Sahlgrenska Hospital, Eastern Hospital, Mölndal Hospital and Högsbo Hospital. Sahlgrenska University Hospital offers employed newly graduated nurses a structured introduction (basic year) with supervision, mentorship and clinical education at two work places (surgical, medical department). The medical department where the study was conducted includes two (medical ward, emergency unit) units.

\section{Design}

This study used content analysis, a qualitative method that involves an inductive approach, to increase the understanding of newly graduated nurses' voices, views and thoughts about their introduction to the nursing profession. This method reveals conflicting opinions and unresolved issues regarding the meaning and use of concepts, procedures and interpretation. Content analysis illustrates the use of several concepts that relate to research procedures to achieve trustworthiness, credibility, dependability and transferability [18,19]. A qualitative research design that relies on trustworthiness, transparency, verification, and reflexivity and that is "information driven" can be helpful when developing insightful and appropriate interpretations within nursing [20]. An inductive methodological approach was used to analyze the data based on the content of newly graduated nurses' thoughts and experiences regarding entering the nursing profession $[18,19]$.

\section{Data collection}

A small convenience sample that is appropriate for qualitative methods was used [20]. The inclusion criteria for participation were registered nurses over the age of 18 years with the ability to understand and speak Swedish and involved the basic year (4-12 month experiences from June 2017-March 2018) in a medical department (medical ward, emergency unit) at Sahlgrenska University Hospital. Two of the authors (EI, JV) sent out an inquiry and an informational letter by email regarding the aim, confidentiality and voluntary participation to all employed newly graduated nurses ( $\mathrm{n}$ $=11$ ) in the department consistent with the inclusion criteria. Two of the authors (El, JV) contacted these nurses $(n=11)$ by text messages (mail, mobile phone) and informed them about the current study. Two reminders (text messages) were sent out, and the first five nurses who were interested in participation 
were included. The ethical guidelines for human and social research were considered throughout the study [21]. The data were collected in March 2018, and the participants $(n=5)$ were informed about the aim and study procedures; confidentiality was assured before the interviews were carried out, which were all conducted in Swedish and started with background questions, including questions regarding age, education and personal experience with healthcare. Furthermore, the data collection focused on five perspectives, namely, the introduction to nursing, supervision, mentorship, work environment and future aspects regarding the introduction to nursing. The interviews started with "Tell me about your experience of introduction at your work place?" Based on the answers, related questions were asked. Examples of situations such as positive and negative aspects of the introduction were explored, and clarifications and further elaborations were made. The interviews lasted between 10 to 25 minutes and were performed individually by two of the authors (EI, $\mathrm{JV})$; all interviews were recorded and then transcribed verbatim. The participants were registered nurses (age 24-42 years) with experience as an RN (1-9 months) and earlier experiences of health care (0-24 years).

\section{Data analysis}

The interviews were analyzed by using manifest qualitative content analysis $[18,19]$ to interpret the meaning from the data content to address trustworthiness [20], with examples drawn from the area of newly graduated nurses' experience of their introduction to nursing in a medical department. Written words were the basis for the analysis, which was performed in the following steps (1-5) which are described in Table 1 as examples of the analysis process: 1 . The transcripts were read and reread to obtain an understanding of and familiarity with the text; 2 . Meaning units (words, sentences or paragraphs) that corresponded to the content areas were selected by using an inductive approach concerning (a) the demands and (b) uncertainty; 3 . Each meaning unit was condensed into a description of its content and labeled with one of 248 codes for example less experienced, limited responsibility; 4. Subcatego- ries were identified and grouped related to the codes; and 5 . One category was identified (managing uncertainty in the nursing profession) and three subcategories were grouped (being humble as a newly graduated nurse, being adaptable as a newly graduated nurse and being a staff member instead of a nursing student) to respond to the experiences of introduction as a newly graduated nurse.

The findings are illustrated with quotes.

\section{Ethical considerations}

Ethical approval and permission for the study were obtained from the manager of the medical department at Sahlgrenska University Hospital and from the firstline managers in the emergency and medical ward (the participants' workplace). No ethical approval was used due to Swedish rules and guidelines regarding student theses and/or projects regarding quality improvement that have no negative effects on participants [21,22]. Respect for the individual nurse was a main concern during the study. All nurses were informed (verbally, in writing) and confirmed by a signed consent form. Moreover, no names were used, and the results are described in categories without identifications. Respect for the participants' integrity and autonomy was thereby shown [21].

\section{Findings}

The data analysis generated one main category, managing uncertainty in the nursing profession, which includes the three subcategories of being humble as a newly graduated nurse, being adaptable as a newly graduated nurse and being a staff member instead of a nursing student. These categories are presented in Figure 1.

\section{Managing uncertainty in the nursing profession}

The main category of managing uncertainty in the nursing profession is described as a transition from nursing student to registered nurse. The newly graduated nurses emphasize the significance of individual support by experienced nurses to manage the demands within the nursing profession and the routines at the

Table 1: Examples of the analysis process.

\begin{tabular}{|c|c|c|c|c|c|}
\hline Meaning unit & Condensation & Code & Domain & Subcategories & Category \\
\hline $\begin{array}{l}\text { So, you were not a perfect } \\
\text { nurse when I walked next } \\
\text { to saying it was like I was a } \\
\text { student in semester } 6 \ldots\end{array}$ & $\begin{array}{l}\text { Not a perfect nurse ... } \\
\text {.. like a student }\end{array}$ & $\begin{array}{l}\text { Less } \\
\text { experienced }\end{array}$ & Demands & $\begin{array}{l}\text { Being humble } \\
\text { as a newly } \\
\text { graduated nurse }\end{array}$ & $\begin{array}{l}\text { Managing } \\
\text { uncertainty in the } \\
\text { nursing profession }\end{array}$ \\
\hline $\begin{array}{l}\text { but I still had to do everything, } \\
\text { but it's always somebody who } \\
\text { controls everything just like } \\
\text { practicing my drug divisions and } \\
\text { everything ... so that, because I } \\
\text { did not have it before, I felt like } \\
\text { a student in one and one half } \\
\text { week... }\end{array}$ & $\begin{array}{l}\text { Do everything, } \\
\text { someone who controls } \\
\text {... felt like a student }\end{array}$ & $\begin{array}{l}\text { Limited } \\
\text { responsibility }\end{array}$ & Uncertainty & $\begin{array}{l}\text { Being adaptable } \\
\text { as a newly } \\
\text { graduated nurse }\end{array}$ & $\begin{array}{l}\text { Managing } \\
\text { uncertainty in the } \\
\text { nursing profession }\end{array}$ \\
\hline
\end{tabular}




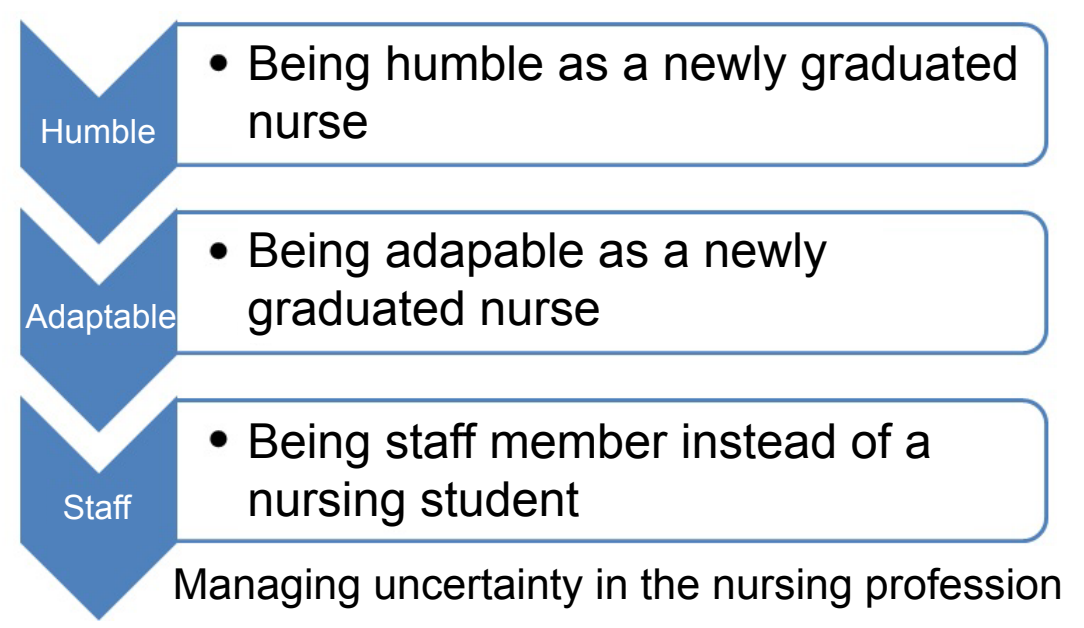

Figure 1: The category and subcategories generated in the results.

work place. The development of nursing skills during the introduction is influenced by the work environment and the specific supervisors' way of facilitate nursing at the ward. Moreover, the participants identify the significance of including organizational perspectives according to the structure of the introduction such as the timetable, tasks regarding nursing responsibility (number of patients) and level of care (complex need) in collaboration with the team. The above factors influence the participants' professional growth such as increased independent work, especially at the emergency unit.

\section{Being humble as a newly graduated nurse}

The subcategory of being humble as a newly graduated nurse is described as coping with the requirements and demands of the nursing profession while being supervised by experienced colleagues. As novices, they emphasize humbleness as crucial to handle the high demands (from themselves and other people), because their skills cannot be compared with an experienced nurse's knowledge and skills:

So, you were not a perfect nurse when I walked next to saying it was like I was a student in semester 6 (last semester within the nursing programme) (Nurse 1)

The introduction ranged between two (emergency unit) to four weeks (medical ward). However, the participants expressed a need for improved reconciliation because the quality of the introduction was influenced by variations of time, personality (supervisor) and workload. They describe that the medical department is labor-intensive, with challenging, complex care situations. The participants indicate the importance of humility in their new occupational role although nursing never becomes perfect. Therefore, they argue that newly graduated nurses need to lower their own demands by reminding themselves that they are novices and cannot be compared with experienced colleagues.

I struggled a lot... over the responsibility that you have to manage as a nurse, I wish to do well and be as good as all those nurses who have worked for several years. I thought that I have to fulfil this entire requirement from day one... that this were the expectations....thankfully I have received a lot of support and feedback. (Nurse 3)

However, the participants describe anxiety from making mistakes due to limited nursing skills that need to be performed in a rapid and changeable work environment with a high workload. Therefore, they identify the significance of individual introduction to their new professional role to ensure patient safety. Being humble is emphasized in a variety of tasks such as patients with a complex diagnosis. The participants address a need for opportunities to discuss the content and assignments with experienced colleagues, which is illustrated as

... maybe it's just that mentoring is very important, I would like to feel like a newly graduated nurse and get support by talking with you, as a mentor. (Nurse 2)

Moreover, the nurses were satisfied with the supervision that they received especially in difficult tasks such as medical devices that they have no previous experience in. Nevertheless, the participants were eager to start working after graduation and indicated that they expect themselves to do their very best. In addition, the participants emphasize the significance of personal responsibility for increased learning in specific situations, which is clarified in the following quote:

I was very excited about this responsibility, and you want to perform and want to be as good as all those nurses who have worked for several years... it's in some way your requirement ... I thought that was what I expected when I worked; however, I have received a lot of support and feedback. (Nurse 3)

Accordingly, the nurses describe the significance of being humble as a newly graduated nurse with the opportunity to influence the introduction of nursing activities and routines at the workplace. 


\section{Being adaptable as a newly graduated nurse}

The subcategory of being adaptable as a newly graduated nurse is described as the conditions for confidence at work supported by a healthy work environment, i.e. helpful and friendly staff members that involved participants in different daily activities. The participants experienced a healthy work environment in terms of limited work tasks provide by the supervisor. To be adaptable as newly graduated nurses is described as being curious and committed in different daily activities that take place at the ward. They stress that staff members encourage them to be involved in different nursing activities, which require adaptation. The nurses felt that it was easy to receive support and respond to questions, which is clarified by the following quote:

"Ask for help 10 times if you do not understand (supervisor)" What do you think about this? You have to ask; otherwise, you get anxiety and very hysterical. (Nurse 1)

The participants emphasize that all staff were helpful regardless of healthcare professionalism. For example, they felt included because their colleagues invited them to participate in various activities at work such as drug administration and demonstrations of new care procedures. One nurse explains the benefit of getting help as

What has been good is that everyone (on the team) is like a tiny supervisor who helps and tells different things. I think it's quite important that all persons join in the introduction ... it is not certain that the supervisor thinks of everything ... then it's good that others jump in and explain how they think, not just the person in charge of the intro; it could be the person next to you ... (Nurse 5)

Being adaptable as a newly graduated nurse was identified as a tool for the best possible outcome regarding the introduction phase. The participants received support from their supervisor according to the introduction plan, which contributed to personal development within nursing. A helpful, supportive and instructive supervisor for specific needs in nursing and the responsiveness of staff members facilitate professional growth in nursing, why adaptation is significant:

but I still had to do everything, but it's always somebody who controls everything just like practicing my drug divisions and everything ... so that, because I did not have it before, I felt like a student in one and one half week (Nurse 2)

Moreover, the participants emphasize that systematic introduction with standardized (nursing activities) and individualized (adaptation to earlier experience) content improves patient safety in the medical department. However, the nurses who were educated outside the local university city indicate their increased need regarding new routines at work, for example, a documen- tation system (medical records) to adapt to. They argue that being adaptable requires improved collaboration (dialogues) between new and trained staff members regarding routines and equipment at work. In addition, the participants were uncertain regarding the content and tasks of a supervisor compared with being a mentor; some participants believed that these were the same, i.e., one person with two different names. The content of mentorship was unclear to them due to unplanned and unorganized meetings during the introduction phase; for this reason, they needed more information regarding how they as newly graduated nurses can use mentors after the introduction in the rest of the basic year. Therefore, being adaptable was accomplished with a healthy work environment.

\section{Being a staff member instead of a nursing student}

The subcategory of being a staff member instead of a nursing student is described as the adaptation to the nursing profession in collaboration with the involved team members, i.e. personal development in being confident as a registered nurse. The participants identify the transition from student to registered nurse as a major step, which is developed during the introduction and the basic year that is included at the two clinical work places. They indicate that they have to manage a gap between nursing education and clinical settings through vocational education including increased nursing responsibility. The participants also address variations in patient responsibility; some nurses well manage the increased patient responsibility due to prior vocational education from earlier personal healthcare experiences, while other nurses state that they were quickly thrown into nursing procedures with many patients to take care of. To improve patient safety, the participants emphasize ongoing dialogue between novice and experienced nurses regarding the responsibility to facilitate the transition to being a staff member. Moreover, these transitions in nursing were delayed due to limited equipment such as the available computers for reading and documenting nursing activities, which is why the participants felt unsure regarding nursing activities concerning medical records.

The nurses' described that the introduction focuses on clinical support; however, they emphasized that mental support was equally important to them to conquer a new working situation with a new professional role as registered nurses. Moreover, barriers such as a shortage of nurses caused problems to the planned introduction, which delays personal development in being confident as a registered nurse. The participants indicate that a high workload among staff members creates uncertainty due to limited possibilities for reflections with colleagues; however, they emphasize that patient safety was always the priority of all nursing activities at the unit. Moreover, the nurses argued that unfamiliar terms (diagnosis, medical records, and treatments) and 
routines influence the transition to be a nurse. Therefore, being supervised in clinical practice was beneficial and increased their knowledge and skills in their daily work. The participants discuss the pros and cons of being taught by different team members as follows:

I thought it might be a bit worse, and then you had to start over every time you got a new supervisor, because they don't really know how much you know or what you have done before. (Nurse 4)

The nurses indicate that high-quality supervision includes one to two supervisors who share the responsibility regarding vocational education, which extends the perspective of newly graduated nurses by showing them different ways of working. Moreover, the participants argue that being a registered nurse is a natural topic to discuss during their mentorship within the basic year in transitioning from novice to expert. In summary, being a staff member was accomplished with supportive teamwork.

\section{Discussion}

The aim of this study was to describe newly graduated nurses' experiences of their introduction to nursing. The main result regarding entering the nursing profession was expressed as managing uncertainty in the nursing profession. Therefore, introduction and mentorship during the first year in nursing are crucial to provide a high quality of care. Newly graduated nurses need support to facilitate their transition from student to registered nurse [23]. According to Benner [5], newly graduated nurses are challenged during their first year in nursing, and theoretical knowledge is performed through behavioral attributes and advanced practice skills. Moreover, Numminen, et al. [24] indicate that the implementation of new evidence-based knowledge starts in nursing education based on the latest science, which is why it is significant that newly graduated nurses are supported at work. However, some limitations involve nursing education (theory versus practice), shortages of nurses' influence, and a high workload with the responsibility for many patients with complex care needs; in this way, nurses' work environment delays the transition into the nursing profession [3,15,25]. In addition, Fukada [26] describes that nursing competency also includes the ability to understand people and healthcare situations by using assessments, communication and clinical judgement. Therefore, nurses need the ability to provide personcentered care in an interprofessional collaboration by focusing on resources and partnerships in health care $[10,27]$. Supervision within the team contributes to safer and less stressed nurses especially when ethical dilemmas are discussed, and professional development influences high-quality nursing. However, Lallo, et al. [9] argue that a high quality of care follows general principles of assessment (standardized) more than research methods (theory in nursing education); for this reason, teaching and educational supervision are considered to be least important among trained staff members.

By using the European Federation of Nurses (EFN) Association guidelines for the implementation and recognition of professional qualifications [7], and the International Council of Nurses' (ICN) Code of Ethics for Nurses [8], the content of the introduction could be standardized in nursing. However, the introduction to nursing also needs to be individualized according to specific nurses' earlier experiences. Therefore, vocational improvement in the basic year for newly graduated nurses is suggested to include supervision (daily/weekly) at the ward by one to two supervisors with team members, regular meetings (weekly/ monthly) with a mentor who works at another ward and group seminars with specific topics that are relevant to the work place (monthly). By using a structured introduction according to the EFN [7] and ICN [8] guidelines combined with a consideration of the specific care unit routines and specific nurses' needs according to their background facilitate a healthy work environment with decreased occupational stress. This approach is consistent with Patterson, et al.'s [12] point of view that enlarging the introduction (time) at the ward level facilitates self-confidence in complex nursing situations, such as drug administration and critical care, and improves nurses' work environment and patient safety [25].

Moreover, experienced nurses improve highquality care by problematizing content and engaging in challenging tasks that facilitate newly graduated nurses to manage new assignments at work in a satisfactory manner [28]. Therefore, an introduction that is led by experienced nurses is crucial during the first year of nursing. Newly graduated nurses need to be challenged. However, being completely prepared in nursing may never occur because new challenges arise within an unpredictable and complex care environment. According to Clipper and Cherry [25], it is common for nurses to make mistakes at the beginning due to a lack of support; therefore, mentoring is a tool for patient safety. This result is consistent with the research's [29-31] explanation that structured mentoring with committed role models (supervisors/mentors) is crucial. Moreover, because of the complexity and variety of health care, nursing education has limitations in preparing for all situations. This finding is consistent with Numminen, et al. [24] who emphasize that newly graduated nurses express anxiety and insecurity due to their expected competence in nursing. Therefore, integrated nursing education that involves a collaboration with university and clinical settings, such as quality improvement and/or a Bachelor's thesis, facilitate evidence-based nursing in relation to lifelong learning for professional development, i.e., theoretical knowledge and practical skills. Therefore, a basic year [32] at the ward level is one 
way to facilitate the transition to become a registered nurse.

The research $[31,33,34]$ shows that mentorship (over time) with trained mentors predicts and prevents mistakes in emergency care; that is, clinical support that uses case management contributes to an improvement in novice nurses' critical thinking. According to Strauss [35] and Wilkinson [36], the introduction develops critical thinking, clinical assessment and prioritization, crucial skills to enable adaptation, reflection and feedback in a new professional role with a high workload, and new routines and skills. To facilitate this transition, support is needed within a healthy work environment [37-40]. This finding is consistent with Benner's [5] idea that vocational education that is performed by helpful and positive colleagues contributes to decreased demands. Teamwork and a healthy work environment require leadership that is approachable, reasonable, and agreeable [41]. When nurse managers use personcenteredness in their leadership [42], it facilitates nurses towards person-centered care [10] and supports health and well-being in every aspect (spirit, body, and mind).

\section{Methodological Considerations}

One limitation of this study could be that it was conducted at one hospital in one country, Sweden. The small sample $(n=5)$ limits the ability to generalize to other settings. However, the trustworthiness of the results was ensured through a scientific systematic analysis that used manifest qualitative content analysis, a well-documented methodology $[18,19]$. This study's validity, however, might be discussed concerning the data collection procedure that involved a limited number of interviewees $(n=5)$ due to limited numbers of available newly graduated nurses at the current medical department. The variations of the nurses' experience of introduction could also be a limitation, which must be considered regarding the transferability of the current study, which is why further studies are needed to develop the knowledge further. However, the area of being a newly graduated nurse is well known, and this research both adds new perspectives and emphasizes earlier results. For further investigation, quantitative research that includes a larger number of participants will contribute to the increased knowledge regarding the transition from nursing student to registered nurse in medical department hospital settings.

\section{Conclusion}

In summary, the introduction to nursing develops theoretical and clinical skills through supervision and mentoring within a team, which reduces the occupational stress among newly graduated nurses. However, there is a lack of evidence regarding the structure of the introduction to nursing. The conclusion of the current study is to combine standardized and individualized content to improve the transition from a novice to an expert nurse. Moreover, mental support, especially during newly graduated nurses' first-year experiences, decreases their anxiety regarding being insufficient and alone at work. Therefore, person-centeredness ensures that the staff (as well as the patients) are capable persons with resources to use in health care such as a healthy work environment.

\section{Conflicts of Interest}

The authors declared no potential conflicts of interest with respect to the research, authorship or publication of this article.

\section{Acknowledgements}

We thank newly graduated nurses at the Medical Department, Sahlgrenska University Hospital who participated in the study and the University of Gothenburg Centre for Person-centered Care (GPCC), Sahlgrenska Academy, University of Gothenburg, Sweden.

\section{References}

1. Mansour M, Mattukoyya R (2018) A cross-sectional survey of british newly graduated nurses' experience of organization empowerment and of challenging unsafe practices. J Contin Educ Nurs 49: 474-481.

2. Vandenkerkhof E, Sears N, Edge DS, Tregunno D, Ginsburg L (2017) Patient safety in practical nurses' education: A cross-sectional survey of newly registered practical nurses in Canada. Nurse Educ Today 51: 48-56.

3. Andersson PL, Edberg AK (2010) The transition from rookie to genuine nurse: Narratives from Swedish nurses 1 year after graduation. J Contin Educ Nurs 41: 186-192.

4. Barnsteiner J, Disch J, Johnson J, Mcguinn K, Chappell K, et al. (2013) Diffusing QSEN competencies across schools of nursing: The AACN/RWJF faculty development institutes. J Prof Nurs 29: 68-74.

5. Benner $P$ (1984) From novice to expert: Excellence and power in clinical nursing practice. Addison-Wesley, Menlo Park, California.

6. Jacobs S (2018) An analysis of the evolution of mentorship in nursing. International Journal of Mentoring and Coaching in Education 7: 155-176.

7. European Federation of Nurses Associations (EFN) (2015) EFN Guideline for the implementation of Article 31 of the Mutual Recognition of Professional Qualifications Directive 2005/36/EC, amended by Directive 2013/55/EU.

8. International Council of Nurses (ICN) (2012) The ICN code of ethics for nurses.

9. Lalloo D, Demou E, Kiran S, Gaffney N, Stevenson M, et al. (2016) Core competencies for UK occupational health nurses: A Delphi study. Occup Med 66: 649-655.

10. Ekman I, Swedberg K, Taft C, Lindseth A, Norberg A, et al. (2011) Person-centered care-ready for prime time. Eur J Cardiovasc Nurs 10: 248-251.

11. Brown RA, Cookes PA (2016) What are the 'necessary' skills for a newly graduating RN? Results of an Australian survey. BMC Nurs 15: 23.

12. Patterson B, Bayley EW, Burnell K, Rhoads J (2010) Orientation to emergency nursing: Perceptions of new 
graduate nurses. J Emerg Nurs 36: 203-211.

13. Delaney C (2003) Walking a fine line: Graduate nurses' transition experiences during orientation. J Nurs Educ 42: 437-443.

14. Dyess SM, Sherman RO (2009) The first year of practice: New graduate nurses' transition and learning needs. J Contin Educ Nurs 40: 403-410.

15. Bennet LL, Grimsley A, Grimsley L, Rodd J (2017) The gap between nursing education and clinical skill. ABNF Journal 28: 96-102.

16. Andersson E, Salickiene Z, Rosengren K (2016) To be involved - A qualitative study of nurses' experiences of caring for dying patients. Nurse Educ Today 38: 144-149.

17. Sahlgrenska University Hospital (2018) About the hospital.

18. Graneheim UH, Lindgren BM, Lundman B (2017) Methodological challenges in qualitative content analysis: A discussion paper. Nurse Educ Today 56: 29-34.

19. Graneheim UH, Lundman B (2004) Qualitative content analysis in nursing research: Concepts, procedures and measures to achieve trustworthiness. Nurse Educ Today 24: 105-112.

20. Polit D, Beck CT (2017) Nursing research: Generating and assessing evidence for nursing practice. Wolters Kluwer Health/Lippincott Williams and Wilkins, Philadelphia.

21. Codex (2017) Rules and guidelines for research. The humanities and social sciences.

22. Swedish Code of Statutes (1982) Health and Medical Services Act.

23. Edwards D, Hawker C, Carrier J, Rees C (2015) A systematic review of the effectiveness of strategies and interventions to improve the transition from student to newly qualified nurse. Int J Nurs Stud 52: 1254-1268.

24. Numminen $O$, Laine $T$, Isoaho $H$, Hupli $M$, Leino-Kilpi $H$, et al. (2014) Do educational outcomes correspond with the requirements of nursing practice: Educators' and managers' assessments of novice nurses' professional competence. Scand J Caring Sci 28: 812-821.

25. Clipper B, Cherry B (2015) From transition shock to competent practice: Developing preceptors to support new nurse transition. J Contin Educ Nurs 46: 448-454.

26. Fukada M (2018) Nursing competency: Definition, structure and development. Yonago Acta Med 61: 1-7.

27. Ricoeur P (1992) Oneself as another. University press, Chicago.

28. Evans J, Boxer E, Sanber S (2008) The strengths and weaknesses of transitional support programs for newly registered nurses. Australian Journal of Advanced Nursing 25: 16-22.

29. Crimlisk JT, Grande MM, Krisciunas GP, Costello KV, Fernandes EG, et al. (2017) Nurse residency program designed for a large cohort of new graduate nurses: Implementation and outcomes. Medsurg Nurs 26: 83-104.

30. Grindel C, Hagerstrom G (2009) Nurses nurturing nurses: Outcomes and lessons learned. Medsurg Nurs 18: 183194.

31. Kuroda T, Kanoya Y, Sasaki AO, Katsuki T, Sato C (2009) Relationship between educational programs offered at midsize hospitals in Japan and novice nurses' anxiety levels. J Contin Educ Nurs 40: 132-138.

32. Sahlgrenska University Hospital (2018) Clinical basic year.

33. Glynn P, Silva S (2013) Meeting the needs of new graduates in the emergency department: A qualitative study evaluating a new graduate internship program. J Emerg Nurs 39: 173178.

34. Harrison-White K, Simons J (2013) Preceptorship: Ensuring the best possible start for new nurses. Nurs Child Young People 25: 24-27.

35. Strauss MB (2009) Easing the transition: A successful new graduate program. J Contin Educ Nurs 40: 216-220.

36. Wilkinson C (2017) Transition to practice. Texas Nursing 91: 12-14.

37. Jones A, Benbow J, Gidman R (2014) Provision of training and support for newly qualified nurses. Nurs Stand 28: 4450 .

38. Moore LW, Leahy C, Sublett C, Lanig H (2013) Understanding nurse-to-nurse relationships and their impact on work environments. Medsurg Nurs 22: 172-179.

39. Mozaffari N, Peyrovi N, Nayeri ND (2015) The social well-being of nurses shows a thirst for a holistic support: A qualitative study. Int J Qual Stud Health Well-being 10: 27749.

40. Rush KL, Adamack M, Gordon J, Lilly M, Janke R (2013) Best practices of formal new graduate nurse transition programs: An integrative review. Int J Nurs Stud 50: 345-356.

41. Nayback-Beebe AM, Forsythe T, Funari T, Mayfield M, Thoms W Jr, et al. (2013) Using evidence-based leadership initiatives to create a healthy nursing work environment. Dimens Crit Care Nurs 32: 166-173.

42. Rosengren K (2016) Person-centred care: A qualitative study on first line managers' experiences on its implementation. Health Services Management Research 29: 42-49. 